\title{
Outcomes of the Special Issue on Extreme and Rogue Waves
}

\author{
E. Pelinovsky ${ }^{1,2}$ and C. Kharif ${ }^{3,4}$ \\ ${ }^{1}$ Dept. of Nonlinear Geophysical Processes, Institute of Applied Physics, Nizhny Novgorod, Russia \\ ${ }^{2}$ Dept. of Information Technologies, National Research University, Higher School of Economics, Nizhny Novgorod, Russia \\ ${ }^{3}$ Institut de Recherche sur les Phénomènes Hors Equilibre, Marseille, France \\ ${ }^{4}$ Ecole Centrale Marseille, Marseille, France
}

\section{Introduction}

The session on "Extreme and Rogue Waves" has been proposed and successfully run for ten years by the Natural Hazard Division of the European Geosciences Union, previously known as the European Geophysical Society. Proceedings of the session were published by Pelinovsky and Kharif (2006, 2008), Kharif et al. (2006) and Pelinovsky et al. (2009). Progresses in this field are further documented by the book Rogue Waves in the Ocean, recently published by Kharif et al. (2009).

The number of extreme waves on the sea surface (freak wave events) is very high. For instance, 79 events were observed during the period 2006-2010 (Nikolkina and Didenkulova, 2011). The accident of the cruise ship "Louis Majesty", on 3 March 2010 near the Spanish coast with two fatalities, was widely described in the mass media. Three people were injured when the ferry "Seastreak" collided with a large wave, on 22 August 2010, in the Atlantic Ocean near the North American coast. The Indonesian region has the largest number of fatalities caused by rogue waves, including several casualties in August 2010 when a ship carrying 60 people (of which only 21 were rescued) capsized and sank following a collision with a freak wave. Two fatalities and ship damage were reported when the super tanker "Aegean Angel" was hit by a large wave off the island of Bermuda, on 30 December 2010.

The prediction of extreme water waves is an important scientific task that requires new theoretical approaches and innovative measurement techniques. Recently, the European Commission has promoted the special project "Extreme Seas" to evaluate the risks related to extreme waves, and many geophysicists and ocean engineers participated in the

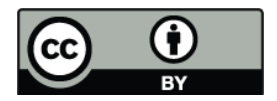

Correspondence to: E. Pelinovsky

(pelinovsky@gmail.com) project. The words "Freak Waves" and "Rogue Waves" are now intensively used in various branches of physics (e.g., nonlinear optics, plasma and super-conductivity), in addition to geophysics (Akhmediev and Pelinovsky, 2010). The growth of research in this field, which is in its "peak of popularity", was demonstrated by the 2011 General Assembly of the European Geosciences Union, where more than 70 papers were presented and discussed in the session on "Extreme Waves".

The 2010 session on "Extreme Waves" held during the General Assembly of the European Geosciences Union, in Vienna, was largely inter-disciplinary. Twenty contributions resulted in papers collected in this special issue. The papers can be loosely grouped in five categories, including: (i) five papers describing empirical observations and related statistics, (ii) eight papers discussing progresses in the numerical simulations of extreme waves, (iii) two papers presenting the results of laboratory experiments aimed at understanding large-amplitude water waves, (iv) three papers discussing large-amplitude waves in internal waters, and (v) two papers dealing with other issues related to freak waves. In the following, we describe briefly the achievements and main results of these papers.

\section{Observations of extreme waves}

Several authors discussed observations of extreme, large amplitude waves. Didenkulova and Anderson (2010) presented a statistical analysis of freak waves measured in the coastal zone of the Baltic Sea ( $2.7 \mathrm{~m}$ in depth) during the period June-July 2008. The dataset lists 97 freak waves had occurred in calm and stormy weather conditions. All of the freak waves were solitary waves, $63 \%$ of them having positive shape, $17.5 \%$ negative shape and $19.5 \%$ a sign-variable shape. It is suggested that the probable mechanism of generation of the freak waves is dispersive focusing. 
Bilyay et al. (2011) presented wave measurements in Filyos, Western Black Sea, Turkey, for a period of two years and at a depth of $12.5 \mathrm{~m}$. There were 209 cases in which the $H / H_{\mathrm{s}}$ ratio was larger than 2.0. The analysis revealed that the distribution of surface profiles of the records containing extreme waves deviates from Gaussian distribution with negative skewness changing between -0.01 and -0.4 , and with high kurtosis in the range from 3.1 to 4.2 .

Liu et al. $(2010,2011)$ have given a very personal view on the occurrence of freak waves on the ocean surface. The authors discussed three separate perspectives: (i) eyewitness accounts, (ii) empirical data, and (iii) theoretical formulation. They further mentioned that the ability to forecast freak waves remains insufficient and proposed a comprehensive freak wave measurement programme to strengthen their understanding.

Gemmrich and Garrett (2011) discussed the probability distribution of data collected in the North Sea. In particular, they conclude that crest height exceedance probabilities may be considerably larger than the statistics obtained from the standard Weibull probability distribution. This should be considered when producing forecasts. Simple Stokes corrections, beyond the second order, are a process that can result in the observed shape of the probability curve. Further investigation of low probability large waves is required.

Vella et al. (2011) described an accumulation of boulders discovered along the rocky coast of the Gulf of Fos, Provence, France, where several mega blocks are scattered behind the coastline, with the largest block ( $33.5 \mathrm{t}$ ) transported $39 \mathrm{~m}$ inland. Along the Mediterranean coasts, the origin of such blocks is attributed to tsunami-generated waves. In the examined case, although the origin is unclear, the differences in surface state between boulders indicate several events generated by extremely large storms. Differences of fauna conservation and surface coloration, suggest that historic storm events have affected these mega-blocks.

\section{Numerical simulations of extreme waves}

Babanin et al. (2011) modelled wave conditions produced by Typhoon Krosa prior to making landfall in Taiwan, on October 2007. For this purpose, the authors used the thirdgeneration wave models SWAN and WWMII. The accuracy of the extreme wave measurement $H_{\max }=32 \mathrm{~m}$, with significant wave height $H_{\mathrm{s}} \sim 24 \mathrm{~m}$ at a depth of $h=38 \mathrm{~m}$ is discussed. It is concluded that the measurement is physically realistic. Further, it is found that neither SWAN nor WWMII are capable to predicting the extreme measurements.

Shemer (2010) studied the Benjamin-Feir instability and evolution of a nonlinear wave train with finite-amplitude sidebands. The application of Benjamin-Feir index (BFI) to evaluate the evolution of wave fields, with non-vanishing amplitudes of sideband disturbances, was investigated using the Zakharov equation as the theoretical model. The analysis considered a three-wave system for which the exact analytical solution of the model equations is available. The author emphasizes that application of the BFI index as a quantitative predictive tool raises questions.

Islas and Schober (2011) analysed the effects of dissipation on the development of rogue waves. The mathematical model used was a new Hamiltonian high-order nonlinear Schrodinger equation recently derived by Gramstad and Trulsen (2011). In the framework of one-dimensional propagation, the authors found that irreversible downshifting occurs when the nonlinear damping is the dominant damping effect. In particular, when only nonlinear damping is present, permanent downshifting occurs and rogue waves do not develop after the downshifting becomes permanent. The generation of rogue waves in the presence of damping for sea states characterised by JONSWAP spectrum is investigated. Present results matched the results of numerical simulations of extended model. Using JONSWAP initial data, it is shown that rogue wave events are well predicted by proximity to homoclinic data.

Touboul and Kharif (2010) investigate the Benjamin-Feir instability in the framework of the two-dimensional, fully nonlinear potential equations, modified to include wind forcing and viscous dissipation. The wind model complies with the Miles' theory. The introduction of dissipation in the equations is briefly discussed. It is found that the presence of wind forcing promotes the occurrence of a permanent frequency-downshifting, without invoking damping due to breaking wave.

Pelinovsky et al. (2011) analysed one of the possible mechanisms for the emergence of freak waves in deep water, based on the dispersive focusing of unidirectional wave packets. The characteristic lifetime of an abnormal wave in the framework of this mechanism for typical conditions is approximately two minutes. Thus, a rapid effect is difficult to predict, and prepare for. A rogue wave quickly changes its shape from a high ridge to a deep depression.

Sergeeva et al. (2011) studied the appearance of freak waves in shallow water of variable depth in the framework of the variable-coefficient Korteweg-de Vries equation. The characteristic wave height varies with depth according to Green's law, and this follows rigorously from the theoretical model. Skewness and kurtosis are computed and it is shown that they increase when the water depth decreases, and simultaneously the wave state deviates from Gaussian. The probability of large-amplitude (rogue) waves increases within the transition zone.

Dao et al. (2011) showed that the numerical method of Smoothed Particle Hydrodynamics (SPH) is capable of reproducing correctly extreme waves and their breaking process. The computational methodology and numerical results are presented and discussed.

$\mathrm{Hu}$ et al. (2011) used the Computational Fluid Dynamics (CFD) flow code AMAZON-SC to study wave loading on a wave energy converter device in heave motion. Results 
include free surface elevations, vertical displacement of the float, induced vertical velocity and heave force for a typical Bobber geometry with a hemispherical base under extreme wave conditions. The method can be used to study the dynamics of the floating bodies in extreme free surface waves.

\section{Laboratory experiments with large-amplitude water waves}

Toffoli et al. (2011) discussed laboratory experiments performed to study the dynamics of three-dimensional mechanically generated waves propagating over an oblique current in partial opposition. The flow velocity varied along the mean wave direction of propagation, with an increasing trend between the wave-maker and the centre of the tank. Tests with regular wave packets traversing the area of positive current gradient showed that the concurrent increase of wave steepness triggered modulational instability on otherwise stable wave trains, and induced the development of very large amplitude waves. In random directional wave fields, the presence of the oblique current resulted in a weak reinforcement of wave instability with a subsequent increase of the probability of occurrence of extreme events. This seems to compensate partially the suppression of strongly non-Gaussian properties due to directional energy distribution.

Jarry et al. (2011) executed experiments in a laboratory tank to study the wave amplification and phase evolution due to a submerged mound. In addition to the classical surface wave measurements, the experimental study takes advantage of photographs to underline crest re-organization above and down-wave the shoal. In particular, together with wave amplification up to more than twice the incident wave, a wave steepening is observed in certain conditions in both the wave direction and in the cross-section. Physical aspects are discussed through the analysis of the diffraction effects on the wave properties.

\section{Large-amplitude waves in internal waters}

Kurkina and Talipova (2011) attempted to explain the appearance of very large amplitude internal waves in the vicinity of the Spitsbergen Island, Barentz Sea. For this purpose, they used the numerical model of the Euler 2-D equations for incompressible stratified fluid to simulate the generation of internal waves by the barotropic tide. It is shown that intensive nonlinear internal waves with amplitudes up to $50 \mathrm{~m}$, and lengths of about 6-12 km, are generated in this zone. The total height of the waves is very large and they must be considered as a significant factor controlling the environment in this basin.

Gavrilov et al. (2011) presented the results of laboratory experiments with large-amplitude internal waves in a twolayer fluid. A mathematical model describing the propagation and decaying of large internal waves in a two-layer fluid is introduced. This model is a variant of the Choi-Camassa equations, with hydrostatic pressure distribution in one of the two layers. It is shown that the numerical scheme developed for the Green-Naghdi equations in open channel flows may be applied to the description of large amplitude internal waves over a shelf.

Makarenko and Maltseva (2011) focused on the mechanism of generation of large-amplitude internal waves due to the interaction of mean flow with submarine ridges. The authors suggest a semi-analytical model of stratified flow over the obstacle under the assumption of small topographic amplitude. Attention is focused on stationary wave patterns forced above the rough terrain. Wave solutions corresponding to sinusoidal topography with a finite number of peaks are calculated and examined.

\section{Associated problems}

Dubard and Matveev (2011) discussed the mathematics of rogue waves in the framework of the nonlinear Schrodinger equation (NLS). The authors construct a multi-parametric family of quasi-rational solutions of the NLS equation, presenting a profile of multiple rogue waves. These solutions have been used to construct a large family of smooth, real, localized rational solutions of the Kadomtsev-Petviashvili equation (KP-I). From the point of view of the Geosciences, NLS equations are relevant to the description of surface waves in deep water, and the KP-I equation occurs in the description of capillary-gravity waves on a liquid surface. In addition, there are many equations of physical importance with their origin in, for example, fibre optics, hydrodynamics and plasma physics, which are gauge equivalent to the NLS equation or to the KP-I equation.

Odent et al. (2010) discussed rogue waves in optical systems, demonstrating wide distribution of rogue wave phenomenon discovered early for huge sea waves. The authors studied pattern formation in an optical system composed of a Kerr medium subjected to optical feedback, in a regime very far from the modulational instability threshold. In this highly nonlinear regime, the dynamics are turbulent and the associated one-dimensional patterns depict rare and intense localized optical peaks. It is shown that their probability density functions have a long tail, indicating the occurrence of rogue events.

Acknowledgements. We would like to thank the journal editors, Fausto Guzzetti and Stefano Tinti, for their support and encouragement, and the editorial staff at Copernicus Gesellschaft $\mathrm{mbH}$ for their professional support. 


\section{References}

Akhmediev, N. and Pelinovsky, E.: Discussion \& Debate: Rogue Waves - Towards a unifying concept? Eur. Phys. J.-Spec. Top., $185,2010$.

Babanin, A. V., Hsu, T.-W., Roland, A., Ou, S.-H., Doong, D.-J., and Kao, C. C.: Spectral wave modelling of Typhoon Krosa, Nat. Hazards Earth Syst. Sci., 11, 501-511, doi:10.5194/nhess11-501-2011, 2011.

Bilyay, E., Ozbahceci, B. O., and Yalciner, A. C.: Extreme waves at Filyos, southern Black Sea, Nat. Hazards Earth Syst. Sci., 11, 659-666, doi:10.5194/nhess-11-659-2011, 2011.

Dao, M. H., Xu, H., Chan, E. S., and Tkalich, P.: Numerical modelling of extreme waves by Smoothed Particle Hydrodynamics, Nat. Hazards Earth Syst. Sci., 11, 419-429, doi:10.5194/nhess11-419-2011, 2011.

Didenkulova, I. and Anderson, C.: Freak waves of different types in the coastal zone of the Baltic Sea, Nat. Hazards Earth Syst. Sci., 10, 2021-2029, doi:10.5194/nhess-10-2021-2010, 2010.

Dubard, P. and Matveev, V. B.: Multi-rogue waves solutions to the focusing NLS equation and the KP-I equation, Nat. Hazards Earth Syst. Sci., 11, 667-672, doi:10.5194/nhess-11-667-2011, 2011.

Gavrilov, N., Liapidevskii, V., and Gavrilova, K.: Large amplitude internal solitary waves over a shelf, Nat. Hazards Earth Syst. Sci., 11, 17-25, doi:10.5194/nhess-11-17-2011, 2011.

Gemmrich, J. and Garrett, C.: Dynamical and statistical explanations of observed occurrence rates of rogue waves, Nat. Hazards Earth Syst. Sci., 11, 1437-1446, doi:10.5194/nhess-11-14372011, 2011.

Gramstad, O. and Trulsen, K.: Hamiltonian form of the modified nonlinear Schrodinger equation for gravity waves on arbitrary depth, J. Fluid Mech., 670, 404-426, 2011.

Hu, Z. Z., Causon, D. M., Mingham, C. G., and Qian, L.: Numerical simulation of floating bodies in extreme free surface waves, Nat. Hazards Earth Syst. Sci., 11, 519-527, doi:10.5194/nhess11-519-2011, 2011.

Islas, A. and Schober, C. M.: Rogue waves and downshifting in the presence of damping, Nat. Hazards Earth Syst. Sci., 11, 383-399, doi:10.5194/nhess-11-383-2011, 2011.

Jarry, N., Rey, V., Gouaud, F., and Lajoie, D.: Gravity wave amplification and phase crest re-organization over a shoal, Nat. Hazards Earth Syst. Sci., 11, 789-796, doi:10.5194/nhess-11-789-2011, 2011.

Kharif, C., Pelinovsky, E., and Tinti, S. (Eds.): Extreme Sea Waves, Special Issue, Nat. Hazards Earth Syst. Sci., http://www. nat-hazards-earth-syst-sci.net/special_issue58.html, 2006.

Kharif, C., Pelinovsky, E., and Slunyaev, A.: Rogue Waves in the Ocean, Springer, 216 pp., 2009.

Kurkina, O. E. and Talipova, T. G.: Huge internal waves in the vicinity of the Spitsbergen Island (Barents Sea), Nat. Hazards Earth Syst. Sci., 11, 981-986, doi:10.5194/nhess-11-981-2011, 2011.
Liu, P. C., Wu, C. H., Bechle, A. J., MacHutchon, K. R., and Chen, H. S.: Brief communication "What do we know about freaque waves in the ocean and lakes and how do we know it?", Nat. Hazards Earth Syst. Sci., 10, 2191-2196, doi:10.5194/nhess-102191-2010, 2010.

Liu, P. C.: Corrigendum to Brief communication "What do we know about freaque waves in the ocean and lakes and how do we know it?" published in Nat. Hazards Earth Syst. Sci., 10, 2191-2196, 2010, Nat. Hazards Earth Syst. Sci., 11, 331-332, doi:10.5194/nhess-11-331-2011, 2011.

Makarenko, N. I. and Maltseva, J. L.: Interference of lee waves over mountain ranges, Nat. Hazards Earth Syst. Sci., 11, 27-32, doi:10.5194/nhess-11-27-2011, 2011.

Nikolkina, I. and Didenkulova, I.: Rogue waves in 2006-2010, Nat. Hazards Earth Syst. Sci., 11, in press, 2011.

Odent, V., Taki, M., and Louvergneaux, E.: Experimental spatial rogue patterns in an optical feedback system, Nat. Hazards Earth Syst. Sci., 10, 2727-2732, doi:10.5194/nhess-10-27272010, 2010.

Pelinovsky, E., Grimshaw, R., and Didenkulova, I. (Eds.): Extreme surface and internal waves, Special Issue, Nonlin. Processes Geophys., http://www.nonlin-processes-geophys.net/ special_issue106.html, 2009.

Pelinovsky, E. and Kharif, Ch.: Rogue Waves, Eur. J. Mech. B.Fluid., 25, 5, 2006.

Pelinovsky, E. and Kharif, C.: Extreme Ocean Waves, Springer, 196 pp., 2008.

Pelinovsky, E., Shurgalina, E., and Chaikovskaya, N.: The scenario of a single freak wave appearance in deep water - dispersive focusing mechanism framework, Nat. Hazards Earth Syst. Sci., 11, 127-134, doi:10.5194/nhess-11-127-2011, 2011.

Sergeeva, A., Pelinovsky, E., and Talipova, T.: Nonlinear random wave field in shallow water: variable Korteweg-de Vries framework, Nat. Hazards Earth Syst. Sci., 11, 323-330, doi:10.5194/nhess-11-323-2011, 2011.

Shemer, L.: On Benjamin-Feir instability and evolution of a nonlinear wave with finite-amplitude sidebands, Nat. Hazards Earth Syst. Sci., 10, 2421-2427, doi:10.5194/nhess-10-2421-2010, 2010.

Toffoli, A., Cavaleri, L., Babanin, A. V., Benoit, M., BitnerGregersen, E. M., Monbaliu, J., Onorato, M., Osborne, A. R., and Stansberg, C. T.: Occurrence of extreme waves in threedimensional mechanically generated wave fields propagating over an oblique current, Nat. Hazards Earth Syst. Sci., 11, 895903, doi:10.5194/nhess-11-895-2011, 2011.

Touboul, J. and Kharif, C.: Nonlinear evolution of the modulational instability under weak forcing and damping, Nat. Hazards Earth Syst. Sci., 10, 2589-2597, doi:10.5194/nhess-10-25892010, 2010.

Vella, C., Demory, F., Canut, V., Dussouillez, P., and Fleury, T. J.: First evidence of accumulation of mega boulders on the Mediterranean rocky coast of Provence (southern France), Nat. Hazards Earth Syst. Sci., 11, 905-914, doi:10.5194/nhess-11-905-2011, 2011. 